\title{
Image Dehazing Based on Haziness Analysis
}

\author{
Fan $\mathrm{Guo}^{1,2} \quad$ Jin Tang ${ }^{1} \quad$ Zi-Xing Cai ${ }^{1}$ \\ ${ }^{1}$ School of Information Science and Engineering, Central South University, Changsha 410083, China \\ ${ }^{2}$ Hunan Engineering Laboratory for Advanced Control and Intelligent Automation, Changsha 410083, China
}

\begin{abstract}
We present two haze removal algorithms for single image based on haziness analysis. One algorithm regards haze as the veil layer, and the other takes haze as the transmission. The former uses the illumination component image obtained by retinex algorithm and the depth information of the original image to remove the veil layer. The latter employs guided filter to obtain the refined haze transmission and separates it from the original image. The main advantages of the proposed methods are that no user interaction is needed and the computing speed is relatively fast. A comparative study and quantitative evaluation with some main existing algorithms demonstrate that similar even better quality results can be obtained by the proposed methods. On the top of haze removal, several applications of the haze transmission including image refocusing, haze simulation, relighting and 2-dimensional (2D) to 3-dimensional (3D) stereoscopic conversion are also implemented.
\end{abstract}

Keywords: Image dehazing, haziness analysis, retinex theory, veil layer, haze image model, haze transmission.

\section{Introduction}

The quality of photograph in our daily life is easily undermined by the aerosols suspended in the medium, such as dust, mist, or fumes. This has an effect on the image, e.g., contrasts are reduced and the surface color becomes fait. Such degraded photographs often lack visual vividness and offer a poor visibility of the scene contents. The goal of haze removal algorithms is to enhance and recover the detail of the scene from haze image. There are many circumstances that accurate haze removal algorithms are needed. In computer vision, most automatic systems for surveillance, intelligent vehicles, object recognition, etc., assume that the input images have clear visibility. However, this is not always true in bad weather. In consumer photography, the presence of fog will be an annoyance to the images for it reduces the contrast significantly. In aerial photography and satellite remote sensing, the photos are much more easily plagued by aerosols ${ }^{[1]}$.

Because of the importance of the haze removal algorithm, much work has been done. These methods can be classified into two main categories: 1) image enhancement based on image processing, and 2) image restoration based on physical model. The classical methods for image enhancement are histogram equalization, homomorphic filter ${ }^{[2]}$, curvelet transform ${ }^{[3]}$, retinex algorithm ${ }^{[4,5]}$, luminance and contrast transform, etc. The image restoration methods focus on the degradation process of the hazed image, aim at establishing the degradation model, deducing the degradation process and compensating the distortion during the degradation to get the undisturbed original image or its best estimation. Compared with the image enhancement methods, restoration methods usually get much natural results, while maintaining detailed information as much as possible

\footnotetext{
Manuscript received September 15, 2012; revised April 25, 2013

This work was supported by National Natural Science Foundation of China (Nos. 91220301, 61175064 and 61273314), Postdoctoral Science Foundation of Central South University (No.126648), New Teacher Fund for School of Information Science and Engineering, Central South University (No. 2012170301)
}

to obtain the ideal haze removal performance. The algorithms belonging to this kind are mostly based on polarizing filter ${ }^{[6,7]}$, user interaction ${ }^{[8,9]}$, known 3-dimensional (3D) models $^{[10]}$ or multiple images ${ }^{[11,12]}$. Although these methods can significantly enhance the visibility, the userinteraction or strict requirement on the inputs limits their applications. Recently, haze removal from a single image has made great progress. Many dehazing algorithms ${ }^{[13,14]}$ based on single image have been developed since Fattal ${ }^{[15]}$. All these works are based on one or more reasonable assumptions, physically or empirically.

Fattal's work $^{[15]}$ was based on the assumption that the transmission and the surface shading are locally uncorrelated. Under this assumption, Fattal estimated the albedo of the scene and then inferred the medium transmission. This approach is physically sound and can produce impressive results. However, the method fails when handling heavy haze images.

Tan's work ${ }^{[13]}$ was based on the observations that the clear-day images have higher contrast compared with the input haze image and he removed the haze by maximizing the local contrast of the restored image. The visual results are visually compelling but may not physical valid.

He et al. ${ }^{[14]}$ proposed the dark channel prior to solve the single image defogging problem. The prior is based on the observation that most local patches in haze-free outdoor images contain some pixels which have very low intensities in at least one color channel. Using this prior, estimated transmission map and the value of atmospheric light can be obtained. For a better purpose, soft matting is used for the estimated transmission map. Combined with the haze image model, a good haze-free image can be recovered by this approach.

In this paper, we propose two image dehazing methods based on haziness analysis. One algorithm regards haze as the veil layer, and the other takes haze as the transmission. The former uses the illumination image obtained by retinex algorithm and the depth information of the original image to remove the veil layer. The latter employs guided filter to 
obtain the refined haze transmission and separate it from the original image. These new algorithms may enhance or restore images from the perspective of haziness with low computation cost and without reference image.

The remaining of this paper is organized as follows. Section 2 describes the haze veil and haze transmission we use in this paper. In Sections 3 and 4, the details of our image dehazing algorithm with haze veil or haze transmission are explained. In Section 5, we extend our algorithms to processing haze video, and some additional applications based on our transmission are also included. A comparative study and quantitative evaluation with other algorithms are shown in Section 6. Finally, we discuss our limitations and conclude the paper in Section 7.

\section{Haziness analysis}

\subsection{Haze veil}

The original idea of image dehazing with haze veil lies in the retinex theory, which deals with compensation for illumination effects in images. The primary work is to decompose a given image $S$ into two different images, the reflectance image $R$, and the illumination image $L$, such that at each point $(x, y)$ in the image domain, $S(x, y)=$ $R(x, y) \cdot L(x, y)$. The goal of the retinex theory is to obtain the reflectance image $R(x, y)$ from the input image $S(x, y)$ by removing effects of the illumination image $L(x, y)$. Based on the retinex theory, the illumination component is estimated by applying smoothing to the haze image, and then the uniform haze veil can be gained by computing the mean of the illumination component. However, according to the Koschmieder model ${ }^{[16]}$, the apparent luminance of the scene object at a different distance is different, so a different haze veil should be assigned to a different position. Thus, the uniform veil is multiplied by the original image to obtain the depth-like map. Considering that the intensity of an image reflects the amounts of photons received by every position of an image, the further the distance between the scene points and camera, the smaller the intensity. Thus, the haze veil reflected by the depth-like map can be measured by intensity, so the intensity component of the depth-like map is extracted to produce the final haze veil associated with different positions.

\subsection{Haze transmission}

The haze image model (also called image degradation model), proposed by McCartney ${ }^{[17]}$ in 1975 , consists of a direct attenuation model and an air light model. The direct attenuation model describes the scene radiance and its decay in the medium, while the air light results from previously scattered light. The formation of a haze image model is as follows:

$$
I(x)=A \rho(x) t(x)+A(1-t(x))
$$

where $I$ is the observed intensity and also the input haze image, $J$ is the scene radiance and also the restored hazefree image, $A$ is the global atmospheric light, and $t$ is the transmission map. Theoretically, the goal of haze removal is to recover $A, \rho(x)$, and $t(x)$ from $I(x)$. Transforming (1), we get

$$
1-\frac{I(x)}{A}=t(x)(1-\rho(x))
$$

where $1-\rho(x)$ is called inverse-albedo. Let $C(x)=1-\rho(x)$, which changes correspondingly to albedo. We further define $B(x)=1-I(x) / A$; equation (2) is transformed as follows:

$$
B(x)=t(x) C(x) .
$$

After obtaining the atmospheric light $A, B(x)$ can be calculated from $I(x)$. Thus, the dehazing issue becomes separating the transmission $t(x)$ and inverse-albedo $C(x)$ from $B(x)$. Here, $B(x)$ describes the ratio of light that human eye does not capture, as shown in Fig. 1 (a). Fig. 1 (b) is the transmission $t(x)$, which describes the portion of the light that is not scattered but reaches the camera. Fig. 1 (c) is the inverse-albedo, which describes the ratio of light that is absorbed by the scene objects. Therefore, $B(x)$ can be seen as the product of transmission and inverse-albedo. This process is similar to the retinex algorithm mentioned above, and we can regard transmission as illuminance and inversealbedo as reflectance of a scene. The transmission is a continuous function of depth. Although there are discontinuities in the depth map, most of the time the variations of the depth are $\operatorname{smooth}^{[1,13]}$, the same for the variations of transmission. Thus, the variations of transmission are largescale. Since the haziness is the main reason for the largescale intensity variation of haze-free image, the following assumption is reasonable: large-scale intensity variations in $B(x)$ are due to the transmission that reflects the density of haziness.

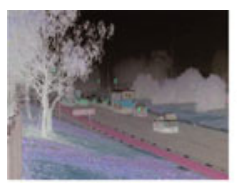

(a)

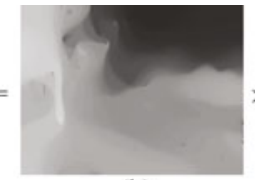

(b)

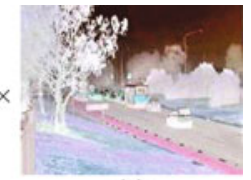

(c)
Fig. 1 Image decomposition

\section{$3 \quad$ Image dehazing with haze veil}

\subsection{Algorithm flow}

Specifically, the proposed veil algorithm has two steps to remove haze from a single image: the first one is to separate the illumination image from the original image and obtains its haze veil together with its own depth information. The second step is to compute the reflectance image by subtracting the haze veil from the original image in the logarithmic domain. The goal of our algorithm is to eliminate the effect of haze veil from the original image then increase the visibility, and finally, enhance contrast by applying adaptive contrast stretching. The flowchart of our method is depicted in Fig. 2.

\subsection{Veil estimation and scene enhance- ment}

The key of the proposed algorithm is how to estimate the haze veil. This process is carried out from red (R), green 
$(\mathrm{G})$, blue (B) three color channels of the original image, separately. Define $F(x, y)$ to be Gaussian with the standard derivation $\sigma$, which is a typical low-pass smoothing function. Firstly, the degraded image is convoluted with the smoothing function. The process can be expressed as follows

$$
\begin{aligned}
& \hat{L}(x, y)=I(x, y) * F(x, y) \\
& F(x, y)=K \mathrm{e}^{-\frac{\left(x^{2}+y^{2}\right)}{\sigma^{2}}}
\end{aligned}
$$

where "*" denotes the convolution operation, $K$ is the normalized factor, $\sigma$ is the standard deviation and controls the degree of blurring. Assuming that the function uses a $w \times w$ window, we determine $K$ satisfying the constraint that makes the sum of $F(x, y)$ equals one. For all $(x, y)$, in order to obtain the haze veil $\tilde{V}(x, y)$ associated with different positions, we first generate the uniform haze veil by computing the mean of the illumination image $\hat{L}(x, y)$, that is, $\bar{L}(x, y)$.

$$
\bar{L}(x, y)=\frac{1}{H W} \sum_{x=1}^{H} \sum_{y=1}^{W} \hat{L}(x, y) .
$$

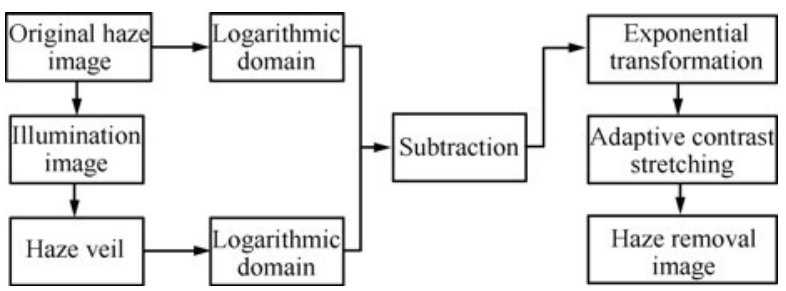

Fig. 2 Flowchart of the algorithm

Note that we can only regard $\bar{L}(x, y)$ as our haze veil when the haze is uniform. If not so, the color of the haze removed image will be distorted seriously. Thus, our algorithm multiplies the uniform veil by the original image to obtain the depth-like map, and the process can be written as

$$
L^{\prime}(x, y)=255-I(x, y) \cdot \bar{L}(x, y) .
$$

Finally, we transform image $L^{\prime}(x, y)$ from the RGB to the $\mathrm{YCbCr}$ color space, and extract the intensity component of image $L^{\prime}(x, y)$, that is, $\tilde{L}(x, y)$, which is our final haze veil. And then, we subtract $\tilde{L}(x, y)$ from the original image $I(x, y)$ in the logarithmic domain, and then we obtain the reflectance image $\tilde{R}(x, y)$ by using exponential transformation.

Fig. 3 illustrate the process described above. Fig. 3 (a) shows the original haze image. Figs. 3 (b) and (c) illustrate the haze veil and the reflectance image, respectively. From Fig. 3 (c), we can see that reflectance image seems too dark, so the post-processing of image enhancement is needed, such as dynamic range compression or histogram equalization. Our algorithm uses adaptive contrast stretching ${ }^{[18]}$ to enhance contrast. Fig. 3 (d) shows our final enhanced results.

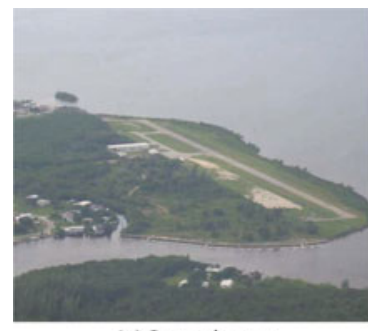

(a) Input image

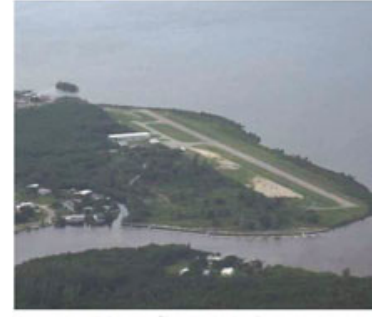

(c) Reflectance image

Fig. 3 Illustration of our method

\section{Image dehazing with transmission}

\subsection{Algorithm flow}

Given a haze image, we first estimate the atmospheric light value $A$ according to the distinctive features of sky region, and also calculate $B(x)$. Next, we estimate the initial haze transmission by using the dark channel prior proposed by He et al. ${ }^{[14]}$. Then we use guided filter ${ }^{[19]}$ to obtain the refined haze transmission $t(x)$, and also calculate $C(x)$ by using (3). As a consequence, the albedo $\rho(x)=1-C(x)$ can be obtained. Finally, the output haze-free image can be gained by multiplying albedo $\rho(x)$ by atmospheric light $A$ according to the haze image model. This process is also depicted in Fig. 4.

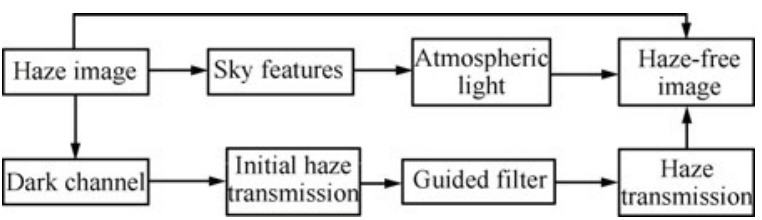

Fig. 4 Haze removal process for video

\subsection{Atmospheric light estimation}

Estimating atmospheric light $A$ should be the first step to restore the haze image. The disturbing factors of white objects lead to an incorrect $A$. Thus, we obtain the value of $A$ by using the distinctive features of sky region: 1) bright minimal dark channel, 2) flat intensity, and 3) upper position. For the first feature, the pixels that belong to the sky region should satisfy $I_{\min }(x)>T_{v}$, where $I_{\min }(x)$ is the dark channel and $T_{v}$ is $95 \%$ of the maximum value of $I_{\min }(x)$. For the second feature, the pixels should satisfy the constraint $N_{\text {edge }}(x)<T_{p}$, where $N_{\text {edge }}(x)$ is the edge ratio map and $T_{p}$ is the flatness threshold. Due to the third feature, the sky region can be determined by searching for the first connected component from top to bottom. Thus, the atmospheric light $A$ is estimated as the maximum value of the corresponding region in the haze image $I(x)$. 


\subsection{Haze transmission estimation and scene restoration}

The transmission map is calculated based on the dark channel prior proposed by He et al. ${ }^{[14]}$. For the haze image, we first estimate the initial haze transmission $\tilde{t}(x)$ :

$$
\tilde{t}(x)=1-\omega \min _{c \in r, g, b}\left(\min _{y \in \Omega(x)}\left(\frac{I^{c}(y)}{A^{c}}\right)\right)
$$

where $\omega$ is a constant parameter for adjusting the amount of haze for distant objects. The value of $\omega$ is application-based and suggested to be $0.95^{[14]}$.

Then, we use the guided filter ${ }^{[19]}$ algorithm to refine the initial haze transmission $\tilde{t}(x)$. For the haze image, it is assumed that refined haze transmission is a linear transform of the haze (guidance) image $I(x)$ in a window $\omega_{x}$ centered at pixel $x$,

$$
\hat{t}(y)=a_{x}^{\mathrm{T}} I(y)+b_{x}, \quad \forall y \in \omega_{x}
$$

where $a_{x}$ and $b_{x}$ are linear coefficients assumed to be constant in $\omega_{x}$. To make the difference between the output $\hat{t}(x)$ and the input $\tilde{t}(x)$ as small as possible, we minimize the following cost function in the local window $\omega_{x}$ centered at pixel $x$,

$$
E\left(a_{x}, b_{x}\right)=\sum_{y \in \omega_{x}}\left(\left(a_{x}^{\mathrm{T}} I(y)+b_{x}-\tilde{t}(x)\right)^{2}+\varepsilon a_{k}^{2}\right) .
$$

The small variable $\varepsilon$ is a regulation parameter to prevent $a_{x}$ from being too large. The solution to (10) is given by

$$
\begin{aligned}
& a_{x}=\left(\sum_{x}+\varepsilon U\right)^{-1}\left(\frac{1}{|\omega|} \sum_{y \in \omega_{x}} I(x) \tilde{t}(x)-u_{x} \bar{t}(x)\right) \\
& b_{x}=\bar{t}(x)-a_{x}^{\mathrm{T}} u_{k}
\end{aligned}
$$

where $\sum_{x}$ is a $3 \times 3$ covariance matrix of $I(x)$ in $\omega_{x}, U$ is a $3 \times 3$ identity matrix, $\bar{t}(x)$ is the mean value of the input $\tilde{t}(x)$ in window $\omega_{x}$, and $u_{k}$ is the mean vector of $I(x)$ in window $\omega_{x}$. By substituting (11) into (9), we get the refined haze transmission $\hat{t}(x)$ for the image. The result is shown in Fig. 5.

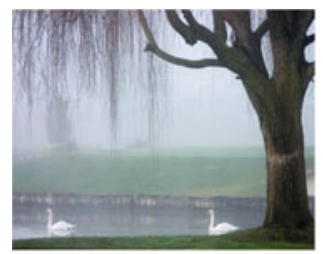

(a) Original haze image

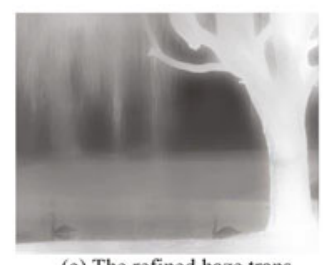

(c) The refined haze trans mission using guided fifter

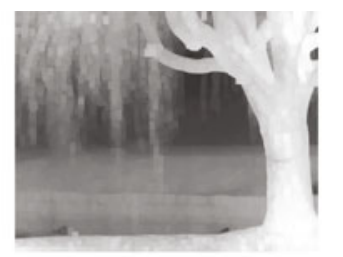

(b) The initial haze transmission

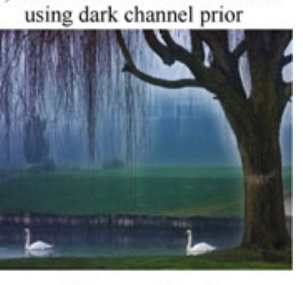

(d) Restored result
Fig. 5 Haze transmission refinement

After obtaining the atmospheric light $A$, we can calculate $B(x)$ combined with input image $I(x)$. Then, $C(x)$ can be also calculated with the haze transmission obtained by our method. The image of $C(x)$ corresponds to the result that eliminates the haziness $t(x)$ from the $B(x)$ image. Thus, the final scene radiance $J(x)$ is recovered by

$$
J(x)=A(1-C(x)) .
$$

Since we already know the inverse-albedo $C(x)$ and the atmospheric light $A$, we can take these values into (12) to obtain the final restored haze-free image $J(x)$. Fig. 5 (c) is the refined haze transmission estimated from color image Fig. 5 (a) using our approach. Fig. 5 (d) is our recovered scene radiance.

\section{Extension to video and application}

\section{$5.1 \quad$ Video dehazing}

The transportation industry would benefit from automatic dehazing technology, especially the video surveillance system. For this application, the primary interest is enhancing video - not single images. For this purpose, our methods should enhance full-resolution video in real-time with a single haze veil or haze transmission.

For the surveillance camera system, the camera is fixed and often positioned high in the air, so the background of each frame is unchangeable and the difference in veil or transmission between a foreground object and the background behind it is usually small. Thus, our method applies the single haze veil or transmission to a series of video frames. During this time, haze veil or transmission is only calculated for the background of the video, in order to apply a universal veil or transmission to more frames with a tolerable error. For simplicity, we define the static part of the scene as the background part and the moving objects in the scene as the foreground part. The background image can be obtained by using the frame differential method. Then, our method estimates the haze veil or transmission of the background image by using the algorithms mentioned above, and applies the same veil or transmission to a series of video frames to obtain the restored images, as shown in Fig. 6. Generally, no significant errors will be induced into the restored image. However, there still exist some estimation errors caused by various background appearances. Therefore, we have to make a trade off between performance and quality.

\subsection{Applications}

After having the refined haze transmission $\hat{t}(x)$, we can add more visual effect on the haze-free image, such as refocusing, haze simulation, relighting and 2D to 3D stereoscopic conversion. Fig. 7 shows the additional effects on the haze-free results.

\section{$6 \quad$ Experimental results}

\subsection{Qualitative comparison}

Our proposed haze veil and haze transmission algorithms can work well for a wide variety of haze images. In the experiments, we performed the algorithms by executing Matlab on a PC with $3.00 \mathrm{GHz}$ Intel Pentium Dual-Core Processor. 

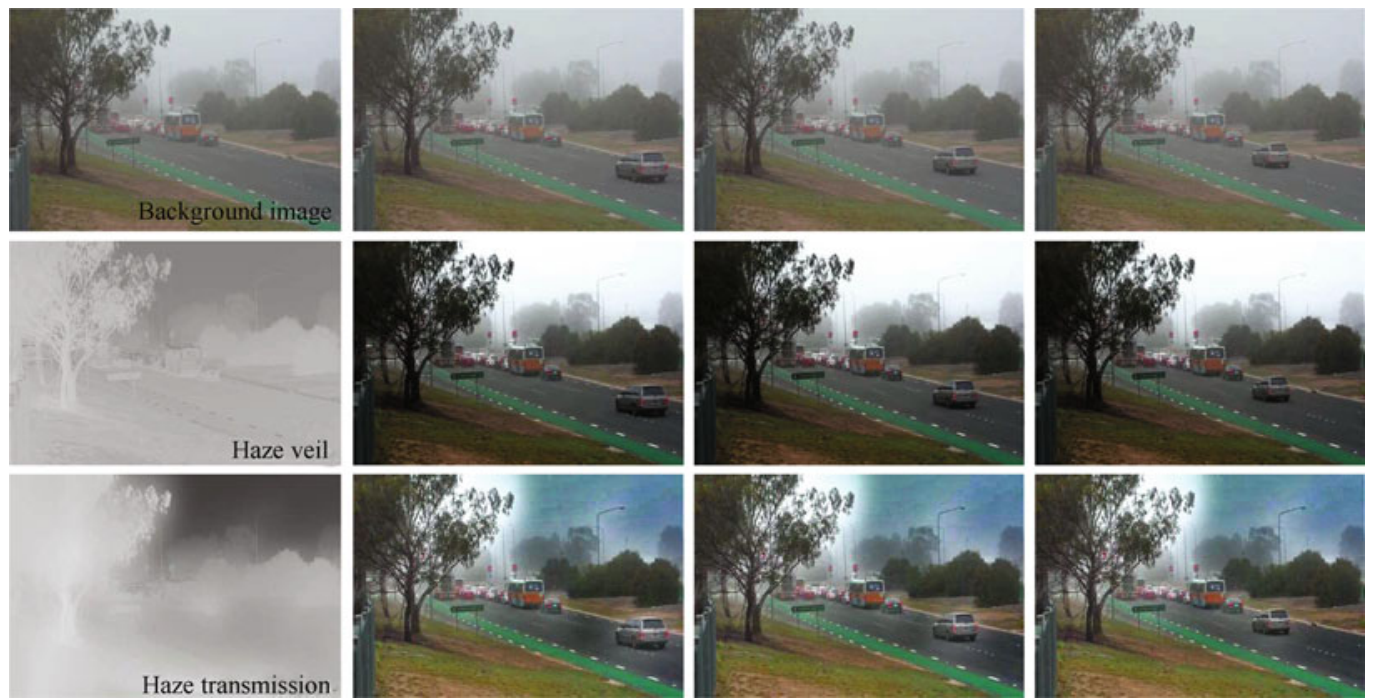

Fig. 6 Our video result. First row: estimated background image and three original frames from video. Second row: universal haze veil and the enhanced frames obtained by using the same veil. Third row: universal haze transmission and the restored frames obtained by using the same transmission
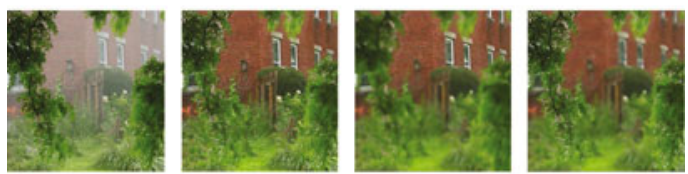

(a) Refocused images. From left to right: input image, recovered image, focus at background red house, and focus at foreground trees
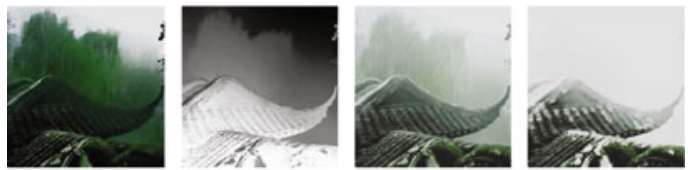

(b) Simulated haze images. From left to right: input image, haze transmission, and simulated haze images with different parameters
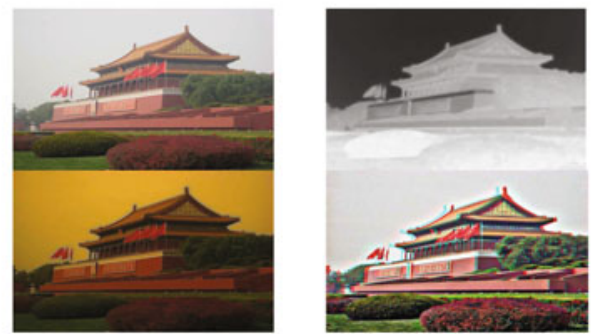

(c) Relighting and 2D to 3D stereoscopic conversion. From left to right: input image, haze transmission, rosy dawn result and anaglyph image

Fig. 7 Additional application based on the haze transmission

Fig. 8 shows a comparison between results obtained by Fattal $^{[15]}$ and our haze veil algorithm. As can be seen in Fig. 8, the Fattal's method can produce a visually pleasing result. However, the method is based on statistics and requires sufficient color information and variance. When the haze is dense, the color information that needed in Fattal's method is not enough for the method to reliably estimate the transmission. Next, we compared our method with Tan's work ${ }^{[13]}$ in Fig. 9. The colors of Tan's result may sometimes be over saturated or distorted, such as the color of traffic sign in Fig. 9 has changed to orange after haze removal. The reason for that is his algorithm is not physically based and may underestimate the transmission. We also compared our method with $\mathrm{He}^{\prime}$ s work ${ }^{[14]}$ in Fig. 10. The overall result of our algorithm is approximately the same as $\mathrm{He}^{\prime} \mathrm{s}$ algorithm. However, $\mathrm{He}^{\prime}$ s method can achieve a better enhancement effect in the distance for Fig. 10 with a higher time complexity. Fig. 11 shows the dehazing results of our proposed algorithms, where we can see that the contrast and clarity are all enhanced significantly compared with the original input image. Figs. 12 and 13 allow the comparison of our results with three state of the art visibility restoration algorithms: Fattal ${ }^{[15]}, \operatorname{Tan}^{[13]}$ and $\mathrm{He}^{[14]}$. Notice that the results obtained with our algorithm seems visually close to the result obtained by $\mathrm{He}$, with better color fidelity and less halo artifacts compared with Tan. However, we find, depending on the image, each algorithm is a trade-off between color fidelity and contrast enhancement. Results on a variety of haze or fog images show that the veil method can achieve a better enhancement effect when the fog is dense, while the haze transmission method can remove haze from a video much faster by using the universal strategy.
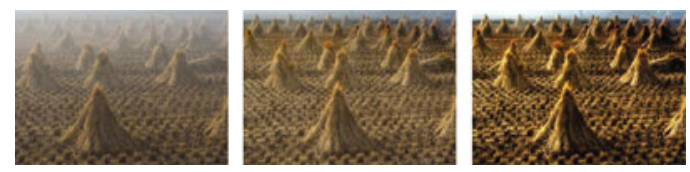

Fig. 8 Comparison with Fattal's work. Left: input image. Middle: Fattal's result. Right: veil method result
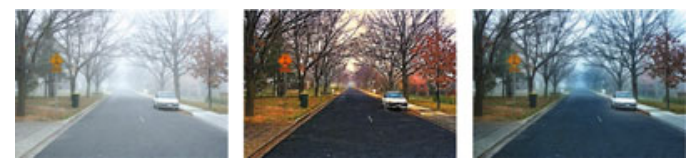

Fig. 9 Comparison with Tan's work. Left: input image. Middle: Tan's result. Right: transmission method result 

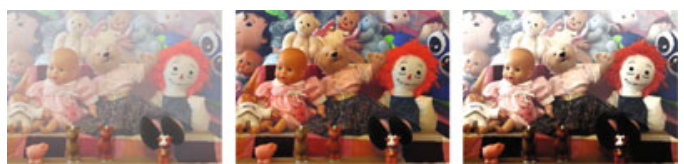

Fig. 10 Comparison with $\mathrm{He}^{\prime}$ s work. Left: input image. Middle: $\mathrm{He}^{\prime}$ s result. Right: veil method result
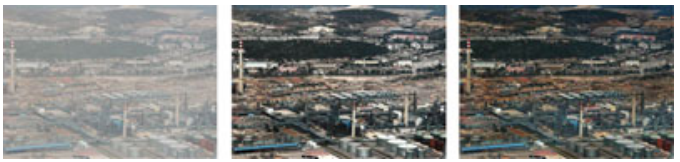

Fig. 11 Our results for comparison. Left: input image. Middle: veil method result. Right: transmission method result
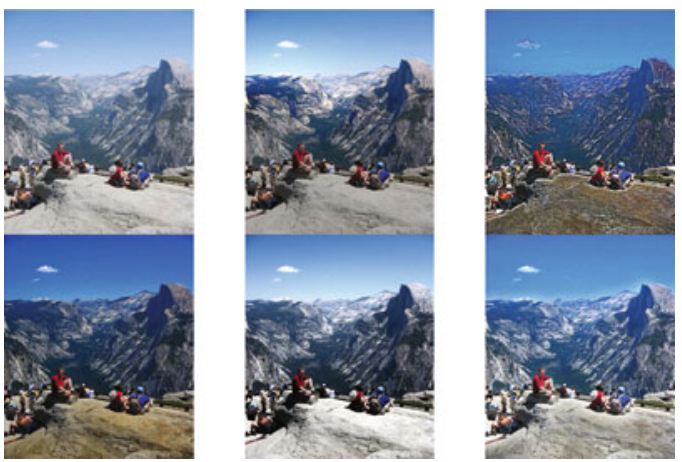

Fig. 12 Experimental results of various dehazing methods. First row: the input image and the results obtained by Fattal and Tan, respectively. Second row: the results obtained by He, our veil method and our transmission method, respectively
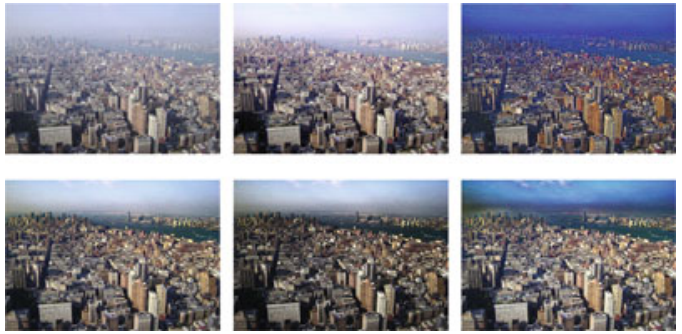

Fig. 13 Experimental results of various dehazing methods. First row: the input image and the results obtained by Fattal and Tan, respectively. Second row: the results obtained by $\mathrm{He}$, our veil method and our transmission method, respectively

\subsection{Quantitative evaluation}

An assessment method dedicated to visibility restoration proposed in [20] is used here to measure the dehazing effect. We first transform the color level image to the gray level image, and use three indicators to compare the two gray level images: the input image and the haze removal image. The visible edges in the image before and after restoration are selected by a $5 \%$ contrast threshold according to the meteorological visibility distance proposed by the international commission of illumination (CIE). To implement this definition of contrast between two adjacent regions, the method of visible edges segmentation proposed in [21] has been used. The computation results of visible edges for our haze veil and haze transmission algorithms on Fig. 12 are given in Fig. 14.

Once the map of visible edges is obtained, we can compute the rate $e$ of edges newly visible after restoration. Then, the mean $\bar{r}$ over these edges of the ratio of the gradient norms after and before restoration is computed. This indicator $\bar{r}$ estimates the average visibility enhancement obtained by the restoration algorithm. At last, the percentage of pixels $\sigma$ which becomes completely black or completely white after restoration is computed. Since the assessment method is based on the definition of visibility distance, the evaluation conclusion which complies with human vision characteristic can be drawn.

These indicators $e, \bar{r}$ and $\sigma$ are evaluated for Fattal ${ }^{[15]}$, $\operatorname{Tan}^{[13]}, \mathrm{He}^{[14]}$ and our method on six images; see Table 1. For each method, the aim is to increase the contrast without losing some visual information. Hence, good results are described by high values of $e$ and $\bar{r}$ and low value of $\sigma$. From Table 1, we deduce that depending on the image, Tan's algorithm generally has more visible edges than our veil, He, our transmission and Fattal algorithms. Besides, we can order the five algorithms in a decreasing order with respect to average increase of contrast on visible edges: Tan, our veil, He, Fattal and our transmission algorithms. This confirms our observations on Figs. 8-13. Table 1 also gives the percentages of pixels becoming completely black or white after restoration. Compared to others, our haze transmission algorithm generally gives the smallest percentage.

Table 1 Comparison with the state of art haze removal algorithms using the three indicators

\begin{tabular}{|c|c|c|c|c|c|c|}
\hline Indicator & $e$ & $\bar{r}$ & $\sigma$ & $e$ & $\bar{r}$ & $\sigma$ \\
\hline Method & Fig. 8 & $(512 \times$ & 384) & Fig. 9 & $\overline{(600 \times}$ & (400) \\
\hline Fattal $^{[15]}$ & 0.209 & 1.274 & 0.078 & 0.416 & 1.149 & 0.490 \\
\hline $\operatorname{Tan}^{[13]}$ & 0.346 & 3.418 & 0.274 & 0.679 & 3.127 & 0.849 \\
\hline $\mathrm{He}^{[14]}$ & 0.287 & 2.399 & 0.008 & 0.532 & 1.607 & 0.073 \\
\hline Haze veil & 0.327 & 2.618 & 0.052 & 0.577 & 2.474 & 0.093 \\
\hline Transmission & 0.214 & 0.984 & 0 & 0.439 & 1.647 & 0 \\
\hline Method & Fig. 10 & $(300 \times$ & 216) & Fig. 11 & $(800$ & 600) \\
\hline Fattal $^{[15]}$ & 0.888 & 3.136 & 0.257 & 0.613 & 2.128 & 0.036 \\
\hline $\operatorname{Tan}^{[13]}$ & 1.374 & 4.715 & 0.539 & 0.716 & 2.537 & 0.092 \\
\hline $\mathrm{He}^{[14]}$ & 1.195 & 2.162 & 0.035 & 0.630 & 2.194 & 0.445 \\
\hline Haze veil & 1.284 & 2.243 & 0.072 & 0.675 & 2.397 & 0.008 \\
\hline Transmission & 1.124 & 1.478 & 0 & 0.625 & 2.127 & 0.004 \\
\hline Method & Fig. 12 & $(576 \times$ & $768)$ & Fig. 13 & (1024 & $\times 768)$ \\
\hline Fattal $^{[15]}$ & 0.058 & 1.203 & 0.149 & 0.106 & 1.535 & 1.699 \\
\hline $\operatorname{Tan}^{[13]}$ & 0.161 & 2.060 & 0.447 & 0.146 & 2.190 & 0.765 \\
\hline $\mathrm{He}^{[14]}$ & 0.141 & 1.329 & 0.160 & 0.123 & 1.629 & 0.014 \\
\hline Haze veil & 0.153 & 1.496 & 0.036 & 0.136 & 1.901 & 0.086 \\
\hline Transmission & 0.113 & 1.186 & 0.009 & 0.150 & 1.281 & 0.012 \\
\hline
\end{tabular}

We also carried out a statistical analysis using Analysis of Variance (ANOVA). ANOVA is a collection of statistical models used to analyze the differences between group means and their associated procedures. Here, we use the statistical method to determine weather the different dehazing algorithms have significant influence on the three indicators. The indicator value of each dehazing algorithm 

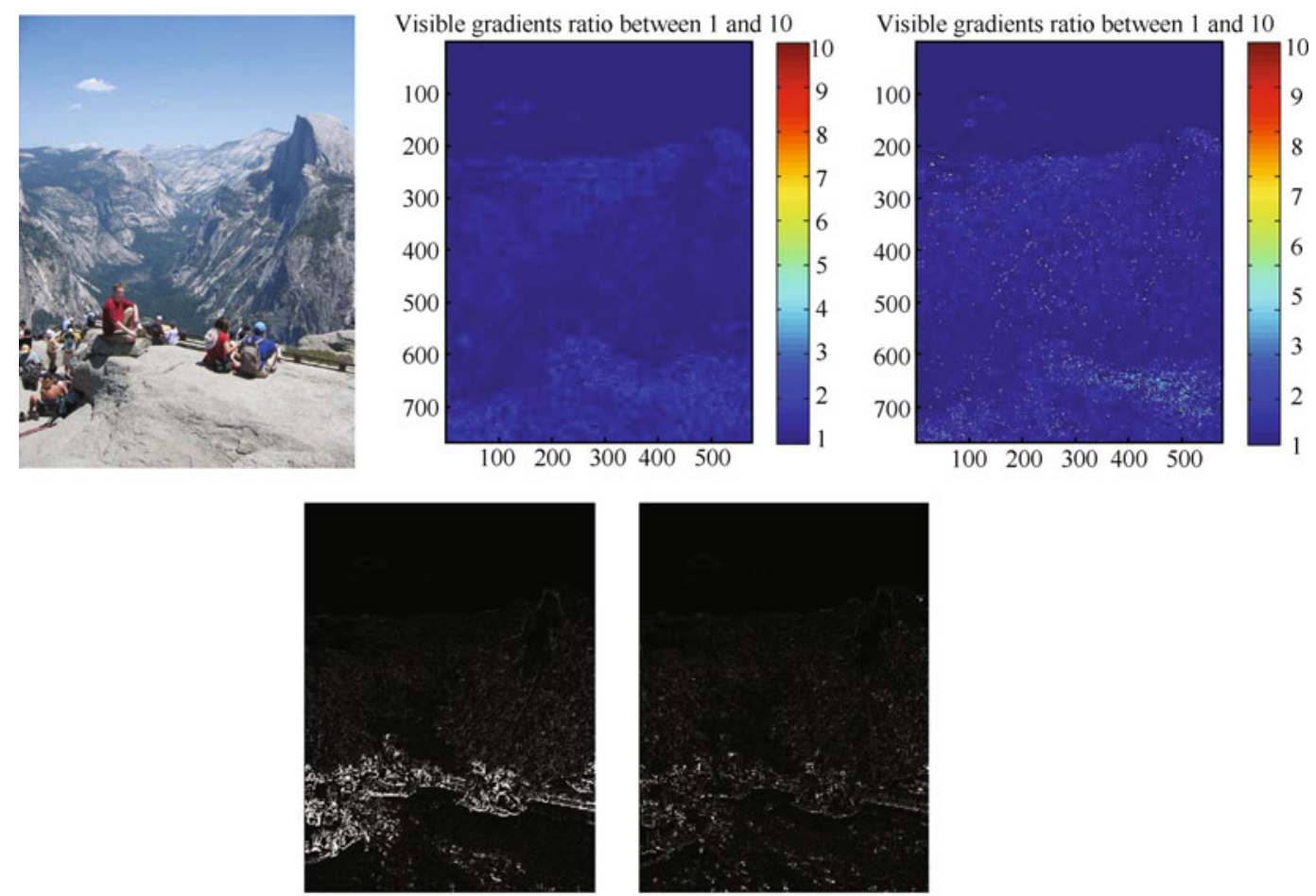

Fig. 14 From left to right: the original image, the map of ratio of the gradients at visible edges for our haze veil and transmission algorithms, the map of visible edge in haze removal image for the two proposed algorithms. The corresponding restored images are shown in Fig. 12

for the six different images were compared by applying one way ANOVA with a 0.05 significance level.

Table 2 shows one way ANOVA results of the indicator data obtained by a software called "OriginPro". When the " $P$ " value in Table 2 is less than 0.05 , there is a significant difference between the groups with a confidence level of $95 \%$. According to this rule, the values of indicator $e$ of different dehazing algorithms were not significantly different, while $\bar{r}$ and $\sigma$ values of different algorithms were significantly different from each other.

Table 2 One way ANOVA results

\begin{tabular}{ccccccc}
\hline Index & Source & $\begin{array}{c}\text { DF } \\
\text { squares }\end{array}$ & $\begin{array}{c}\text { Sum of } \\
\text { square }\end{array}$ & Mean & $F$ & $P$ \\
\hline \multirow{4}{*}{$e$} & Model & 4 & 0.1271 & 0.03177 & 0.19428 & 0.93908 \\
& Error & 25 & 4.08867 & 0.16355 & & \\
& Total & 29 & 4.21577 & & & \\
& Model & 4 & 8.5211 & 2.13028 & 5.09233 & 0.00385 \\
$\bar{r}$ & Error & 25 & 10.45825 & 0.41833 & & \\
& Total & 29 & 18.97935 & & & \\
& Model & 4 & 1.26633 & 0.31658 & 3.09322 & \\
$\sigma$ & Error & 25 & 2.55868 & 0.10235 & 0.03374 & \\
& Total & 29 & 3.82502 & & & \\
\hline
\end{tabular}

This conclusion illustrates that for indicator $e$, different algorithms have no significant influence on it, so the dehazing effect can not be well evaluated by using it. The reason why that some algorithms may increase contrast so strong is that the corresponding haze removal images will have halos near some edges, which make the $e$ value unreliable. Whereas for $\bar{r}$ and $\sigma$, different dehazing algorithms have significant influence on the two indicators. Thus, they can effectively measure the dehazing effect for each algorithm. From Table 1, we can see that compared with other existing algorithms, the proposed algorithms, as the whole, can get a better trade-off between $\bar{r}$ and $\sigma$, which demonstrates that similar or better quality results can be obtained by using our proposed algorithms. This confirms our observations on Figs. 8-13.

\subsection{Computation times}

For an image of size $s_{x} \times s_{y}$, the complexity of the our haze veil algorithm is $\mathrm{O}\left(s_{x} s_{y}\right)$, which implies the complexity of the veil method is a linear function of the number of input image pixels. For the our haze transmission algorithm, the haze removal image can be obtained in $\mathrm{O}(N)$. Next, computational time is considered by testing the state of the art methods in the Matlab environment.

For He's method, its time complexity is relatively high since the matting Laplacian matrix $L$ in the method is so huge that for an image of size $s_{x} \times s_{y}$, the size of $L$ is $s_{x} s_{y} \times s_{x} s_{y}$, so $20 \mathrm{~s}$ is needed to process a 600 -by- 400 pixels image. The computational times of Fattal's and Tan's methods are even longer than He's method. They take about 40 and five to seven minutes to process an image which is of size $600 \times 400$, respectively. Our proposed methods have a relatively faster speed, $2 \mathrm{~s}$ is needed to obtain a haze removal image of the same size by using haze veil method, and $4 \mathrm{~s}$ is needed by using haze transmission method. Fig. 15 shows the computing speeds of the four methods with different image sizes $(128 \times 96,256 \times 192$, $512 \times 384$, and $1024 \times 768$ ). 


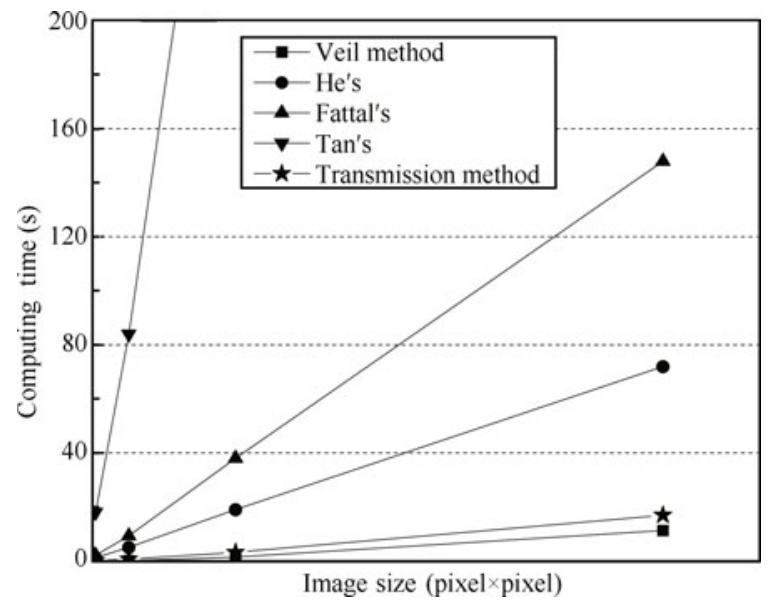

Fig. 15 Comparison of computing speeds

\section{Conclusions}

Image dehazing is an important issue in computer vision. This paper proposes two image dehazing algorithms based on the haziness analysis, which regards haze as the veil layer or take haze as the transmission. Our algorithms produce visually pleasing dehazing results and tend to preserve the main details better than previous techniques. Compared with the state-of-the-art algorithms, our algorithms have two main advantages: 1) no other reference image is needed, and 2) its speed is relatively faster while maintaining comparable performance. A comparative study and quantitative evaluation demonstrate the efficiency of our algorithms. Our algorithms could be further improved by employing some advanced haze image models.

We have shown that we can generate real-time and good haze removal results by regarding haze as the veil layer to be subtracted or as the transmission to be separated from the original haze image. However, for the haze transmission method, it shows a tendency to have halos near some edges. This indicates that although the method can generate a refined haze transmission very fast by using guided filter, it is not an accurate transmission map. This disqualifies our algorithm from dealing effectively with an object without a clear shape, and the haze removal result is sometimes highcolored. Nevertheless, we can improve the overall quality of a haze image by enhancing the main details.

\section{Acknowledgments}

The authors would like to thank Dr. Raanan Fattal, Dr. Robby T. Tan and Dr. Kaiming He for providing their haze removal images. The authors would like to thank the reviewers and the editors for their valuable comments.

\section{References}

[1] J. W. Zhang, L. Li, G. Q Yang, Y. Zhang, J. Z. Sun. Local albedo-insensitive single image dehazing. The Visual Computer, vol. 26, no. 6-8, pp. 761-768, 2010.

[2] M. J. Seow, V. K. Asari. Ratio rule and homomorphic filter for enhancement of digital colour image. Neurocomputing, vol. 69, no. 7-9, pp. 954-958, 2006.
[3] J. L. Starck, F. Murtagh, E. J. Candès, D. L. Donoho. Gray and color image contrast enhancement by the curvelet transform. IEEE Transactions on Image Processing, vol. 12, no. 6, pp. 706-717, 2003.

[4] D. J. Jobson, Z. Rahman, G. A. Woodel. Properties and performance of a center/surround retinex. IEEE Transactions on Image Processing, vol. 6, no. 3, pp. 451-462, 1997.

[5] D. J. Jobson, Z. Rahman, G. A. Woodell. A multiscale retinex for bridging the gap between color images and the human observation of scenes. IEEE Transactions on Image Processing, vol. 6, no. 7, pp. 965-976, 1997.

[6] Y. Y. Schechner, S. G. Narasimhan, S. K. Nayar. Instant dehazing of images using polarization. In Proceedings of 2001 IEEE Computer Society Conference on Computer Vision and Pattern Recognition, IEEE, Piscataway, USA, pp. I325-I-332, 2001

[7] S. Shwartz, E. Namer, Y. Schechner. Blind haze separation. In Proceedings of 2006 IEEE Computer Society Conference on Computer Vision and Pattern Recognition, IEEE, Piscataway, USA, pp. 1984-1991, 2006.

[8] S. G. Narasimhan, S. K. Nayar. Chromatic framework for vision in bad weather. In Proceedings of IEEE Conference on Computer Vision and Pattern Recognition, IEEE, Hilton Head Island, SC, USA, pp. 598-605, 2000.

[9] W. Hu, G. D. Yuan, Z. Dong, X. M. Shu. Improved single image dehazing using dark channel prior. Journal of Computer Research and Development, vol. 47, no. 12, pp. 21322140, 2010.

[10] N. Hautiere, J. P. Tarel, D. Aubert. Towards fog-free invehicle vision systems through contrast restoration. In Proceedings of IEEE Conference on Computer Vision and Pattern Recognition, IEEE, Minneapolis, MN, USA, pp. 1-8, 2007.

[11] S. K. Nayar, S. G. Narasimhan. Vision in bad weather. In Proceedings of the 7th IEEE International Conference on Computer Vision, IEEE, Washington, DC, USA, pp. 820$827,1999$.

[12] G. Chen, T. Wang, H. Q. Zhou. A novel physics-based method for restoration of foggy day images. Journal of Image and Graphics, vol. 13, no. 5, pp. 888-893, 2008. (in Chinese)

[13] R. T. Tan. Visibility in bad weather from a single image. In Proceedings of IEEE Conference on Computer Vision and Pattern Recognition, IEEE, Anchorage, AK, USA, pp. 1-8, 2008.

[14] K. M. He, J. Sun, X. O. Tang. Single image haze removal using dark channel prior. In Proceedings of IEEE Conference on Computer Vision and Pattern Recognition, IEEE, Miami, FL, USA, pp. 1956-1963, 2009.

[15] R. Fattal. Single image dehazing. ACM Transactions on Graphics, vol. 27, no. 3, Article No. 72, 2008.

[16] W. E. K. Middleton. Vision Through the Atmosphere, Canada: University of Toronto Press, pp. 56-58, 1952.

[17] E. J. McCartney. Optics of the Atmosphere: Scattering by Molecules and Particles, New York: John Wiley and Sons, pp. 23-32, 1976

[18] D. B. Xu, C. B. Xiao, J. Yu. Color-preserving defog method for foggy or hazy scenes. In Proceedings of the 4th International Conference on Computer Vision Theory and Applications, INSTICC Press, Lisboa, Portugal, pp. 69-73, 2009. 
[19] K. M. He, J. Sun, X. O. Tang. Guided image filtering. IEEE Transactions on Pattern Analysis and Machine Intelligence, vol. 35, no. 6, pp. 1397-1409, 2013.

[20] N. Hautiere, J. P. Tarel, D. Aubert, E. Dumont. Blind contrast enhancement assessment by gradient ratioing at visible edges. Image Analysis \& Stereology, vol. 27, no. 2, pp. 87-95, 2008.

[21] R. Kohler. A segmentation system based on thresholding. Computer Graphics and Image Processing, vol. 15, no. 4, pp. 319-338, 1981.

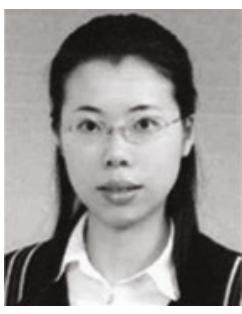

Fan Guo received the B. Sc. degree from the Central South University, China in 2005, M.Sc. and Ph. D. degrees in computer science from Central South University, China in 2008 and 2012, respectively. Currently, she is a postdoctoral fellow at the School of Information Science and Engineering, Central South University.

Her research interests include image proality. cessing, pattern recognition and virtual re-

E-mail: guofancsu@163.com

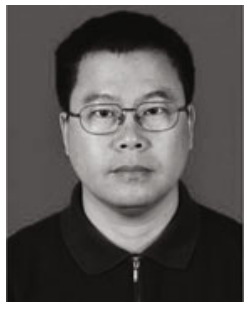

Jin Tang graduated from Peking University, China in 1987. He received the M. Sc. degree from Peking University in 1990 and the Ph. D. degree from Central South University, China in 2002. He is currently a professor at the Laboratory of Intelligence Science and Technology, Central South University.

His research interests include image processing, computer vision and robotics. E-mail: tjin@csu.edu.cn (Corresponding author)

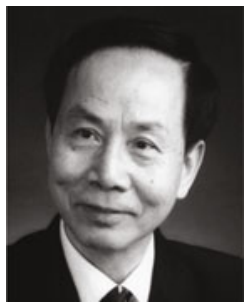

Zi-Xing Cai received the diploma degree from the Department of Electrical Engineering, $\mathrm{Xi}^{\prime}$ an Jiaotong University, China in 1962. He has been teaching and doing research at the School of Information Science and Engineering, Central South University, China since 1962. He has authored/coauthored over 600 papers and 30 books. He received over 30 state, province, and university awards in science, technology, and teaching. One of the most recent prizes is the State Eminent Professor Prize of China.

His research interests include intelligent systems, artificial intelligence, intelligent computation, and robotics.

E-mail: zxcai@csu.edu.cn 